\title{
On material velocities and non-locality in the thermo-mechanics of continua
}

\author{
J.D. Goddard \\ Department of Mechanical and Aerospace Engineering, University of California, San Diego, 9500 Gilman Drive, La Jolla, CA 92093-0411, USA
}

\section{A R T I C L E I N F O}

\section{Keywords:}

Material velocities

Gradient effects

Statistical mechanics

Homogenization

Non-locality

Galilean invariance

Onsager symmetry

\begin{abstract}
A B S T R A C T
Following is a discussion of several issues surrounding the continuum modeling of discrete particulate systems, particularly the uncertainty in the definition of continuum-level material particles and velocities. The work is motivated in part by various proposals for the introduction of supplemental velocity fields into the thermo-mechanics of single-component fluids. A review and modification are given of the relevant continuum field equations, based on the Eulerian rather than the conventional Lagrangian description, and a connection is made to the well-known statistical mechanics of Kirkwood and coworkers for pointparticles.

The Noetherian technique of Green and Naghdi is employed to derive the momentum balance directly from the particle-level energy balance, and it is shown that non-barycentric effects in particle motion engender stress asymmetry. It is conjectured that this and related effects are generally bound up with higher-gradient effects and the breakdown of the simple-material model for continuum thermo-mechanics. To illustrate this, a theory of non-local linear viscoelasticity is presented and compared with Brenner's "bi-velocity hydrodynamics" and Müller's "extended thermodynamics" for heat flux and stress. These models are shown to represent expansions in spatial wave vector, representing a Knudsen number, and temporal frequency, representing a Deborah number. Suggestions are made for further work to explore the associated effects of couple-stress and the consequences of departures of entropy flux from heat flux.
\end{abstract}

(c) 2010 Elsevier Ltd. All rights reserved.

\section{Introduction - Field theoretic background}

The Lagrangian point of view and the underlying notion of a material particle (or point) is firmly imbedded in rational continuum mechanics and the associated virtual-work principles [38,36,11,6,37], although the precise physical meaning of such particles is sometimes ambiguous. More often than not, they are barycentric points, associated with mass and the conservation of mass, although mass plays no role in fields such as elastostatics.

The issue is relevant to statistical mechanics, where the "homogenization" or "coarse-graining" $[24,13,15]$ of a system of discrete bodies or subparticles, is employed to define an hypothetical overlying continuum. As reflected by the earliest works on solid elasticity [3], the identification of a definite material particle with a definite collection of subparticles (in a "representative material volume element") is relatively straight-forward in the absence of significant thermal motion. By contrast, in fluid-like states of matter, large-scale thermal (or Brownian) motion leads to uncertainty ${ }^{1}$ in this identification, and one may plausibly argue that the continuum-level "material particle" exists only on time scales defined by thermal diffusion.

E-mail address: jgoddard@ucsd.edu

${ }^{1}$ We recall that attempts have been made to attribute quantum-mechanical uncertainty to analogous effects [32]. 
Relevant to this issue is a series of papers by Brenner [1,2, and references therein], indicating that the conventional barycentric velocity is insufficient to describe certain departures from Navier-Stokes theory in non-homogeneous fluids. As a remedy, he proposes a supplementary velocity field, qualified variously by terms such as volume velocity or work velocity, which is subsequently employed in various constitutive equations for diffusive energy flux and stress.

Stimulated in part by Brenner's work, several investigators attempt to identify other relevant velocities based on statistical mechanics. For example, Eu [9], making use of the Irving-Kirkwood [18] formulae discussed below, defines a velocity based on collective modes of molecular motion, but the mechanical consequences and physical measurability are not clear to this author. The position taken here is that physical measurability or observability is an eminently desirable property of constitutive variables and that such measurability is ultimately dependent on some appeal to conservation laws. ${ }^{2}$ For example, the measurability of barycentric velocity, defined as the ratio of local mass flux ${ }^{3}$ to mass density, is guaranteed, at least conceptually, by the conservation of mass. In the absence of some similar principle for non-barycentric "particles" and "velocities", they should be regarded as mathematical artifice, however convenient.

One goal of this article is to show how the uncertain status of various material points and velocities is tied up with highergradient effects and the breakdown of the (Coleman-Noll) model of thermo-mechanically simple materials. Hence, a proper treatment of such effects requires modificaton of the latter, such as the thermodynamics of Müller [26,24, and references therein]. In the two following sections, we review the relevant continuum field equations and their statistical mechanical counterparts, indicating a certain arbitrariness in the concept of material velocity and its consequences for constitutive theories. In the final section, we consider a non-local form of linear viscoelasticity, in order to illustrate the breakdown of the simple-material model and the relevance to the issues discussed above.

\section{Continuum fields and balances}

In contrast to the conventional continuum mechanics, and in the spirit of [26], we employ spatial (Eulerian) forms, rather than integrals on a manifold of material particles. As made evident below, this formulation makes for easier comparison to the standard versions of statistical mechanics.

Thus, the local balance of an extensive tensor-valued quantity $\mathbf{A}$, endowed with spatial density field ${ }^{4}$ $\varrho_{\mathbf{A}}(\mathbf{x}, t)\left[=\varrho_{\mathbf{A}}^{i_{1}, \ldots, l_{n}}(\mathbf{x}, t)\right]$, flux $\boldsymbol{\varphi}_{\mathbf{A}}(\mathbf{x}, t)\left[=\varphi_{\mathbf{A}}^{i_{1}, \ldots, i_{n+1}}(\mathbf{x}, t)\right]$ and production rate $\gamma_{\mathbf{A}}(\mathbf{x}, t) b\left[=\gamma_{\mathbf{A}}^{i_{1}, \ldots, i_{n}}(\mathbf{x}, t)\right]$, is given by:

$$
\partial_{t} \varrho_{\mathbf{A}}+\nabla \cdot \boldsymbol{\varphi}_{\mathbf{A}}=\gamma_{\mathbf{A}}
$$

such that ${ }^{5}$

$$
\mathbf{A}(V, t)=\int_{V} \varrho_{\mathbf{A}}(\mathbf{x}, t) d V(\mathbf{x})
$$

is the amount of $\mathbf{A}$ contained in the spatial region $V$. The notation conveys the point that tensor densities such as $\varrho=\varrho_{\mathbf{A}}$ inherit their tensorial rank from the superscripted quantity $\mathbf{A}$, whereas spatial fluxes $\boldsymbol{\varphi}$ are one rank higher.

The balance (1) with $\gamma_{W}=0$ applies to an arbitrary conserved scalar $\mathbf{A}=W$, which defines a tracer-velocity field $\mathbf{u}(\mathbf{x}, t)=\boldsymbol{\varphi}_{W} / \varrho_{W}$ and the associated tracer-particle field $\mathbf{x}_{W}(\mathbf{x}, t)$ satisfying the partial differential equation ${ }^{6}$

$$
\partial_{t} \mathbf{x}_{W}(\mathbf{x}, t)+\mathbf{u} \cdot \nabla \mathbf{x}_{W}(\mathbf{x}, t)=\mathbf{0}
$$

whose characteristic curves $d \mathbf{x}_{W} / d t=\mathbf{u}\left(\mathbf{x}_{W}, t\right)$ define tracer-particle paths.

The extended thermodynamics of Müller and coworkers [26], which provides a useful conceptual backdrop to the present work, involves a hierarchy of balances of the form (1) based loosely on the kinetic theory of gases. The kinetic theory also dictates the form of their constitutive closures, ${ }^{7}$ which involve weakly non-local forms of the type discussed below in Section 4 . As indicated by the following analysis, the balances in their hierarchy cannot be regarded as completely independent.

We focus on thermo-mechanical quantities $\mathbf{A}$ whose spatial dependence is inherited from the generalized kinematics $\mathbf{v}, \theta$ of a material body occupying $V$, including a barycentric velocity field $\mathbf{v}(\mathbf{x}, t)$ and an additional set of variables $\theta(\mathbf{x}, t)$, provisionally interpreted as one or more frame-indifferent scalar "temperatures". We denote the corresponding functionals by:

$$
\psi(\mathbf{x}, t)=\psi[\mathbf{v}, \theta](\mathbf{x}, t), \quad \text { where } \psi=\underline{\varrho}, \boldsymbol{\varphi}, \quad \text { or } \gamma,
$$

\footnotetext{
2 The tortuous history of Newton's "force" in classical mechanics is a prime example, providing much of the motivation for Lagrangian mechanics and related virtual-work principles. By means of the latter, one defines force in terms of an exchange of (conserved) energy accompanying changes in configuration, which elegantly defers the problem to one of defining configuration.

${ }^{3}$ This runs counter to numerous textbook definitions of mass flux in terms of a previously undefined velocity. It also points up a fundamental difficulty with non-conserved entities such as entropy, which we generally cannot collect and store in a given volume of space and, conversely, cannot completely capture from a given material flow.

${ }^{4}$ Here and in the following, components of a tensor are specified in square brackets, along with the defining equal sign.

5 For the sake of brevity, we denote a quantity and its measure by the same symbol.

6 Lamb [23, Chapter 1], noting that these equations apply to the labeling of any "particle", traces them through Stokes to Lagrange.

7 Which are simply algebraic connections between various fields and their partial derivatives, to which one cannot readily assign rôles of "dependent" vs. "independent" variables; e.g. [26, Eq. (2.13)] gives various components of stress and heat flux in terms of the derivatives of these quantities.
} 
and these are assumed to depend on $\mathbf{v}\left(\mathbf{x}^{\prime}, t^{\prime}\right), \theta\left(\mathbf{x}^{\prime} t^{\prime}\right)$ for $\mathbf{x}^{\prime} \in V, t^{\prime}<t$. While mass density $\rho(\mathbf{x}, t)$ might also be included in the list of field variables, we prefer to treat it as derivable from $\mathbf{v}(\mathbf{x}, t)$ via the mass balance, which as shown below follows from the energy balance. The rôle of mass density will be taken up again in Section 4, where we specialize to a weakly non-local theory in which the above functionals depend on a limited number of spatial gradients $\mathbf{v}, \theta, \nabla \mathbf{v}, \nabla \theta, \nabla \nabla \mathbf{v}, \nabla \nabla \theta$, etc.

By means of the Noetherian technique of Green and Naghdi [16] all the standard balances of continuum mechanics follow from the form invariance of the energy balance under change of frame. ${ }^{8}$ Whereas their treatment is based on integral balances over bodies, the same results follow from the stronger local form (1) employed here, whenever the fields are sufficiently smooth or, otherwise, whenever appropriate discontinuity conditions are provided.

\subsection{Key balances and fluxes}

To motivate the following treatment, we note that, within the present framework and with the provisional assumption of simple materials, the energy balance (with $\mathbf{A} \stackrel{\text { def }}{=} E$ ) can be written as

$$
\begin{aligned}
& \boldsymbol{\varphi}_{E}=\varrho_{E} \mathbf{v}+\mathbf{q}-\mathbf{T} \mathbf{v}=\varrho_{E} \mathbf{v}+\mathbf{j}_{E}-\mathbf{T u}, \quad \gamma_{E}=\varrho \mathbf{b} \cdot \mathbf{v}+r, \quad \text { with } \\
& \mathbf{j}_{E}=\mathbf{q}+\mathbf{T}(\mathbf{u}-\mathbf{v}), \quad \varrho_{E}=\rho\left(\frac{1}{2} v^{2}+\varepsilon\right), \quad \rho=\varrho_{M} .
\end{aligned}
$$

Here, $\varepsilon$ denotes specific internal energy, q conventional heat flux, T Cauchy stress, b barycentric body force, and $r$ the radiant energy input. Also, we distinguish the barycentric velocity $\mathbf{v}$ from a dynacentric velocity $\mathbf{u}$, playing the same role in the energy balance as Brenner's work velocity [2] which he occasionally associates with a "diffuse volume flux" $\mathbf{j}_{w}=\mathbf{u}-\mathbf{v}$, about which more will be said later.

Since $\mathbf{u}-\mathbf{v}$ is by definition Galilean invariant, so is the diffuse energy flux $\mathbf{j}_{E}$ defined in (5). ${ }^{9}$ Nothing is gained at this juncture by the introduction of distinct conjugate stresses, say, $\mathbf{T}_{\mathbf{v}}, \mathbf{T}_{\mathbf{u}}$, since the associated power flux can be written as

$$
\mathbf{T}_{\mathbf{v}} \mathbf{v}+\mathbf{T}_{\mathbf{u}} \mathbf{u}=\mathbf{T v}+\mathbf{T}_{\mathbf{u}}(\mathbf{u}-\mathbf{v}), \quad \text { where } \mathbf{T}=\mathbf{T}_{\mathbf{v}}+\mathbf{T}_{\mathbf{u}},
$$

which merely changes the definition of $\mathbf{j}_{E}$.

Since $\mathbf{T}$ is assumed to be Galilean-invariant, a Galilean transformation of (5), i.e. a shift of $\mathbf{v}$ by a constant velocity $\boldsymbol{v}$, adds terms to $\varrho_{E}$ that are both linear and quadratic in $v$. According to Green and Naghdi [16], these lead, respectively, to the energy and mass balances, and their treatment is now adapted to the present formulation.

\subsubsection{Changes of frame}

As a variant on the standard representation of a change of frame

$$
\mathbf{x}^{+}=\mathbf{Q}(t) \mathbf{x}+\xi(t), \quad \mathbf{Q}^{-1}=\mathbf{Q}^{T},
$$

by the finite Euclidean group, we express it here in terms of the infinitesimal generators, $\delta \boldsymbol{\Theta}=-\delta \boldsymbol{\Theta}^{T}=(\delta \mathbf{Q}) \mathbf{Q}^{-1}$ and $\delta \boldsymbol{v}$, with

$$
\begin{aligned}
& \delta \mathbf{x}=(\delta \boldsymbol{\Theta}) \mathbf{x}+\delta \boldsymbol{\xi}, \quad \delta \mathbf{v}=(\delta \boldsymbol{\Theta}) \mathbf{v}+(\delta \boldsymbol{\Omega}) \mathbf{x}+\delta \boldsymbol{v}, \quad \delta \theta=0, \\
& \delta \partial_{t}=-[(\delta \boldsymbol{\Omega}) \mathbf{x}+\delta \boldsymbol{v}] \cdot \nabla, \quad \delta \nabla=(\delta \boldsymbol{\Theta}) \cdot \nabla, \quad \text { where } \\
& \delta \boldsymbol{v}=\delta \partial_{t} \boldsymbol{\xi}, \quad \text { and } \delta \boldsymbol{\Omega}=\delta \partial_{t} \boldsymbol{\Theta},
\end{aligned}
$$

Then, with

$$
\mathfrak{D}(\cdot)=\delta\{(\cdot), \nabla(\cdot), \nabla \otimes \nabla(\cdot), \ldots\}
$$

denoting variation of the underlying vector space, we define an objective $n$ th-rank tensor field $\psi\left[=\psi \psi_{i_{1}, \ldots, i_{n}}\right]=\psi[\mathbf{v}, \theta](\mathbf{x}, t)$ as one that transforms according to

$$
\begin{aligned}
& \delta \psi=\mathcal{L}(\delta \boldsymbol{\Theta}) \psi+\delta_{\mathbf{v}} \psi[\mathbf{v}, \theta \mid \mathrm{D} \mathbf{v}]+\delta_{\theta} \psi[\mathbf{v}, \theta \mid \grave{D} \theta] \text { with } \\
& {[\mathcal{L}(\mathbf{B}) \psi]^{i_{1}, \ldots, i_{n}}=\sum_{m=1}^{n} B_{j_{m}}^{i_{m}} \psi^{i_{1}, \ldots, i_{m-1} j_{m}, i_{m+1} \ldots, i_{n}}}
\end{aligned}
$$

$\mathcal{L}$ denotes the Lie derivative with respect to an $\underset{*}{*}$ rbitrary second-rank tensor $\mathbf{B}$, which is bilinear in $\mathbf{B}$ and $\psi$ and vanishes whenever the latter is a scalar $(n=0)$. Also, $\delta_{\mathbf{v}}\left[\left.(\cdot)\right|^{*}\right]$ and $\delta_{\theta}\left[\left.(\cdot)\right|^{*}\right]$, linear in ${ }^{*}$, represent suitable functional (e.g. Fréchet) derivatives with respect to the arguments $(\cdot)$. Whenever these functional derivatives vanish, the tensor field is frame-indifferent according to the standard definition.

Suppressing notation for the quantity A, we can write the balance (1) as

$$
\mathcal{D}_{t} \varrho+\nabla \cdot \varphi^{\circ}=\gamma^{\circ}
$$

\footnotetext{
${ }^{8}$ Their derivation of the entropy balance appears to involve a physically unacceptable shift in the origin of absolute temperature and will not be employed here.

9 This term "diffuse" is employed in deference to other literature on the subject, whereas "relative" would be a better terminology.
} 
with

$$
\boldsymbol{\varphi}^{\circ}=\boldsymbol{\varphi}-\mathbf{v} \otimes \varrho, \quad \gamma^{\circ}=\gamma-\varrho \nabla \cdot \mathbf{v}-\mathcal{L}(\mathbf{W}) \varrho,
$$

where

$$
\mathcal{D}_{t} \varrho=d_{t} \varrho-\mathcal{L}(\mathbf{W}) \varrho, \quad d_{t}=\left(\partial_{t}+\mathbf{v} \cdot \nabla\right), \quad \mathbf{W}=\frac{1}{2}\left(\nabla \mathbf{v}^{T}-\nabla \mathbf{v}\right)
$$

denote, respectively, the co-rotational derivative, material time derivative, and vorticity. The relation (11) represents one possible invariant form for frame-indifferent tensor fields, an important subclass of which are called Galilean by Müller [26].

\subsubsection{Linear momentum and mass from energy}

In the special case $\mathbf{A}=E$, (11) reduces to

$$
\begin{aligned}
& d_{t} \varrho_{E}+\nabla \cdot \boldsymbol{\varphi}_{E}^{\circ}=\gamma_{E}^{\circ}, \quad \text { where } \\
& \boldsymbol{\varphi}_{E}^{\circ}=\boldsymbol{\varphi}_{E}-\varrho_{E} \mathbf{v}, \quad \gamma_{E}^{\circ}=\gamma_{E}-\varrho_{E} \nabla \cdot \mathbf{v},
\end{aligned}
$$

and the various terms are defined in (5) for simple materials.

Specializing to Galilean transformations, $d \mathbf{\Omega}=\mathbf{0}, \delta \mathbf{v} \equiv \delta \boldsymbol{v}$, independent of $t$, the variational derivative of (14) gives the linear momentum balance:

$$
d_{t} \varrho_{\mathbf{p}}+\nabla \cdot \boldsymbol{\varphi}_{\mathbf{p}}=\gamma_{\mathbf{p}}, \quad \text { where }\left\{\boldsymbol{\varrho}_{\mathbf{p}}, \boldsymbol{\varphi}_{\mathbf{p}}, \gamma_{\mathbf{p}}\right\}=\partial_{\mathbf{v}}\left\{\boldsymbol{\varrho}_{E}, \boldsymbol{\varphi}_{E}, \gamma_{\mathbf{p}}\right\},
$$

with $\boldsymbol{\varphi}_{\mathbf{p}}^{\circ}=\partial_{\mathbf{v}} \boldsymbol{\varphi}_{E}^{\circ}=-\mathbf{T}$ defining the stress tensor.

A further derivative $\partial_{\mathbf{v}}$ of (15) gives a balance for a second-rank mass tensor $\mathbf{M}$ with density $\varrho_{\mathbf{M}}=\partial_{\mathbf{v}} \varrho_{\mathbf{p}}$. However, the further requirement that $\varrho_{E}$ be an isotropic function of $\mathbf{v}$, assumed in (5), gives $\varrho_{\mathbf{M}}=\rho \mathbf{1}$.

By considering the more general Euclidean group (8) one can derive a standard angular momentum balance [16], although the derivation involves well-known assumptions as to the way in which $\varepsilon$ is allowed to depend on acceleration relative to an inertial frame [26,28]. Setting aside this issue, we consider now the connection to the statistical mechanics of discrete particulate systems.

\section{Connection to statistical mechanics}

Homogenization associates a continuous medium with a discrete mechanical system $\mathcal{B}$ consisting of $N$ distinct bodies or particles and described by a discrete set of phase-space coordinates $\mathbf{z}=\left\{\mathbf{x}_{\alpha}, \mathbf{v}_{\alpha}, \ldots\right\} \in \mathcal{Z}$ defining configuration and velocities. ${ }^{10}$ Although not pursued here, the number $N$ should be connected to the representative volume element, which is in turn related to the coarse-graining discussed below.

As a variant on a method that goes back at least as far as Kirkwood and coworkers [18,33,34,27,12,29,13,31,15], ${ }^{11}$ one "smears out" each particle $\alpha=1,2, \ldots, N$, in physical space by means of a weighting or coarse-graining density $n_{\alpha}(\mathbf{x})$, where $\mathbf{x}$ is spatial position. In general, the $n_{\alpha}(\mathbf{x})$ are assumed to be continuous and differentiable in $\mathbf{x}$, up to an order dictated by the continuum model, and to satisfy

$$
\int_{V} n_{\alpha}(\mathbf{x}) d V(\mathbf{x})=1
$$

where $V$ is the spatial region occupied by the model continuum. Given then any extensive quantity defined by a space tensor A of rank $n$ and having assigned $\mathbf{A}_{\alpha}(\mathbf{z})$ with

$$
\mathbf{A}_{\mathcal{S}}=\sum_{\alpha \in \mathcal{S}} \mathbf{A}_{\alpha}
$$

for any subset of particles $\mathcal{S} \subseteq \mathcal{B}$, the associated spatial density is

$$
\varrho_{\mathbf{A}}(\mathbf{x}, t)=\sum_{\alpha} \mathbf{A}_{\alpha} n_{\alpha}, \quad \text { where } \sum_{\alpha}:=\sum_{\alpha \in \mathcal{B}},
$$

whose $t$ dependence arises solely through dependence on $\mathbf{z}$.

The abstract distributions $n_{\alpha}$ take on a concrete significance in the conventional statistical mechanics of discrete particles, where Kirkwood and coworkers [18,13] replace them by the Dirac delta $\delta\left(\mathbf{x}_{\alpha}-\mathbf{x}\right)$ to yield a density operator

$$
P_{\mathbf{A}}(\mathbf{x}, t)=\sum_{\alpha} \mathbf{A}_{\alpha} \delta\left(\mathbf{x}_{\alpha}-\mathbf{x}\right)
$$

\footnotetext{
10 As the obverse of the notational convention of [13], we employ Roman indices for tensor components and Greek for particle enumeration.

11 In the works [12,13], the present author overlooks several important contemporaneous works of Murdoch and Murdoch and Bedeaux cited in [31], which survey much of the same terrain from a different perspective.
} 
where the $\mathbf{x}_{\alpha}$ represent suitably defined particle positions. As singular realizations of the discrete system, such a linear operator is dual to the $N$-particle distributions in phase-space $\mathcal{Z}$, say $f(\mathbf{z}, \mathbf{x}, t)$, defining phase-space averages:

$$
\varrho_{\mathbf{A}}(\mathbf{x}, t)=\left\langle P_{\mathbf{A}}\right\rangle:=\int_{\mathcal{Z}} P_{\mathbf{A}} f(\mathbf{z}, \mathbf{x}, t) d V(\mathbf{z}),
$$

where $d V(\mathbf{z})$ represents an appropriate volume measure in $\mathcal{Z}$.

The Dirac delta is a special case of the more general Galilean-invariant homogenization,

$$
n_{\alpha}(\mathbf{x})=n\left(\left|\mathbf{x}_{\alpha}-\mathbf{x}\right|\right),
$$

where $n$ is independent of $\alpha$. In this sense, (20) represents a natural homogenization of the form

$$
n_{\alpha}(\mathbf{x})=\int_{\mathcal{Z}} n\left(\left|\mathbf{x}_{\alpha}-\mathbf{x}\right|\right) f(\mathbf{z}, \mathbf{x}) d V(\mathbf{z}) .
$$

We set aside questions related to homogenization and averaging in the time domain addressed recently by Murdoch and coworkers [29,31], although these are highly relevant to the issue of thermal fluctuations.

As a departure from previous works [18,27,13], the particle positions $\mathbf{x}_{\alpha}$ are not regarded ab initio as mass centroids. Starting from a more general definition of velocity, the Appendix indicates how (19) leads to the balance:

$$
\partial_{t} P_{\mathbf{A}}+\nabla \cdot \boldsymbol{\Phi}_{\mathbf{A}}=\Gamma_{\mathbf{A}},
$$

with flux and production operators defined, respectively, by

$$
\begin{aligned}
& \mathbf{\Phi}_{\mathbf{A}}=\sum_{\alpha}\left\{\mathbf{h}_{\alpha} \otimes \dot{\mathbf{A}}_{\alpha}+\mathbf{u}_{\alpha} \otimes \mathbf{A}_{\alpha} \delta_{\alpha}\right\} \quad \text { with }, \\
& \mathbf{h}_{\alpha}=\frac{1}{W} \sum_{\beta} \boldsymbol{w}_{\beta} \int_{\mathbf{x}_{\beta}}^{\mathbf{x}_{\alpha}} \delta(\mathbf{y}-\mathbf{x}) d \mathbf{y}, \quad \dot{\mathbf{A}}_{\alpha}=\dot{\mathbf{z}} \cdot \partial_{\mathbf{z}} \mathbf{A}_{\alpha}, \quad \mathbf{u}_{\alpha}=\dot{\mathbf{x}}_{\alpha},
\end{aligned}
$$

and

$$
\begin{aligned}
& \Gamma_{\mathbf{A}}=\overline{\dot{\mathbf{A}}} P_{W}, \quad \text { with } P_{W}=\sum_{\alpha} w_{\alpha} \delta_{\alpha}, \\
& \overline{(\quad)}=\frac{1}{W} \sum_{\alpha}()_{\alpha}, \quad W=\sum_{\alpha} w_{\alpha}, \quad \text { and } \quad \delta_{\alpha}=\delta\left(\mathbf{x}_{\alpha}-\mathbf{x}\right) .
\end{aligned}
$$

The $w_{\alpha}$ represent arbitrary non-negative weights, which are also subject to a balance, with $\mathbf{A} \equiv W$ in (23)-(25). If the weights represent a conserved tracer, with $\dot{w}_{\alpha} \equiv 0$, then they are fit to define a supplemental "material velocity" $\mathbf{u}(\mathbf{x}, t)$. For systems of identical particles with $w_{\alpha}=w$ independent of $\alpha$, we also may take $w=1$ and $W=N$ in the above formulae, but $\mathbf{x}_{\alpha}$ may be distinct from barycentroids, so that $\mathbf{u}_{\alpha} \neq \mathbf{v}_{\alpha}$.

The first term in (24), with $\mathbf{h}_{\alpha}$ involving a path integral of a delta function, ${ }^{12}$ represents one contribution to a diffusive flux relative to a convective flux associated with the second term in (24).

In keeping with (11), it is also convenient to define the supplemental quantities based on barycentric velocity $\mathbf{v}$

$$
\begin{aligned}
& \boldsymbol{\Phi}_{\mathbf{A}}^{\circ}=\boldsymbol{\Phi}_{\mathbf{A}}-\mathbf{v} \otimes P_{\mathbf{A}}, \quad \Gamma_{\mathbf{A}}^{\circ}=\Gamma_{\mathbf{A}}-P_{\mathbf{A}} \nabla \cdot \mathbf{v}-\mathcal{L}(\mathbf{W}) P_{\mathbf{A}}, \\
& \quad \text { where } \mathbf{v}=\boldsymbol{\varphi}_{M} / \rho .
\end{aligned}
$$

Owing to the introduction of continuum-level averages, these quantities represent non-linear operators on the relevant distributions.

With appropriate choices of $\mathbf{A}_{\alpha}$ and with $w_{\alpha}=m_{\alpha}$ the standard barycentric balances follow from (23)-(25) [18,33,29,13]. However, as suggested by the continuum balances given above, the mass and momentum balances follow from the Galilean invariance of the energy balance. ${ }^{13}$ In particular, one obtains the momentum-operator balance from the Lagrangian forms:

$$
\begin{aligned}
& P_{\mathbf{p}}=\partial_{\mathbf{v}} P_{E}=\mathbf{\Phi}_{M}, \quad \mathbf{\Phi}_{\mathbf{p}}^{\circ}=\partial_{\mathbf{v}} \mathbf{\Phi}_{E}^{\circ}, \quad \Gamma_{\mathbf{p}}^{\circ}=\partial_{\mathbf{v}} \Gamma_{E}^{\circ}, \quad \text { with } \\
& \mathbf{p}_{\alpha}=\partial_{\mathbf{v}} E_{\alpha}, \quad \dot{\mathbf{p}}_{\alpha}=\partial_{\mathbf{v}} \dot{E}_{\alpha}
\end{aligned}
$$

As one of the main points of the present work, we note that for a system of identical particles subject to an external body force field $\mathbf{b}_{\alpha}=\mathbf{b}\left(\mathbf{x}_{\alpha}\right)$, the energy balance is given by (23)-(25), with:

$$
\mathbf{A}_{\alpha}=E_{\alpha}=m\left(v_{\alpha}^{2} / 2+\varepsilon_{\alpha}\right), \quad \text { with } \dot{E}_{\alpha}=\mathbf{f}_{\alpha} \cdot \mathbf{v}_{\alpha}+m \mathbf{b}_{\alpha} \cdot \mathbf{v}_{\alpha}+m \dot{\varepsilon}_{\alpha},
$$

where $\mathbf{v}_{\alpha}$ denotes barycentric velocity, with $v_{\alpha}=\left|\mathbf{v}_{\alpha}\right|$, and $\varepsilon_{\alpha}$ represents specific internal energy, including all forms of internal kinetic energy (rotational, vibrational, etc), as well as internal potential energy. Also, $\mathbf{f}_{\alpha}=\sum_{\beta} \mathbf{f}_{\alpha \beta}$ denotes the resultant of

\footnotetext{
${ }^{12}$ Up to an additive solenoidal term $\oint \delta(\mathbf{y}-\mathbf{x}) d \mathbf{y}$, this integral can be reduced to Noll's form [33,13,31] by integration along the straight-line path $\mathbf{y}=s \mathbf{x}_{\alpha}+(1-s) \mathbf{x}_{\beta}, 0 \leqslant s \leqslant 1$.

${ }_{13}$ This runs counter to the chain of reasoning in the works just cited, which derive the energy balance from the mass and momentum balances.
} 
pairwise inter-particle forces $[18,33,13]$, while $\dot{\varepsilon}_{\alpha}$ includes all other forms of energy exchange, including work due to interparticle couples.

If $\mathbf{x}_{\alpha}, \mathbf{u}_{\alpha}$ denote non-barycentric quantities, e.g. position and instantaneous velocity of any center of $\mathbf{f}_{\alpha}$, (any point without moment), then its instantaneous power is given by:

$$
\mathbf{f}_{\alpha} \cdot \mathbf{u}_{\alpha} \equiv \mathbf{f}_{\alpha} \cdot \mathbf{v}_{\alpha}+\mathbf{f}_{\alpha} \cdot\left(\mathbf{u}_{\alpha}-\mathbf{v}_{\alpha}\right),
$$

where the second term, representing particle rotation and deformation, has the same Galilean-invariant form as other terms included in $\dot{\varepsilon}_{\alpha}$ in (28). At any rate, by making use of (23)-(27) one finds after a bit of algebra the operator version of (5):

$$
\boldsymbol{\Phi}_{E}^{\circ}=\Pi \mathbf{v}+\mathbf{Q}=\Pi \mathbf{u}+\mathbf{J}_{E}, \quad \mathbf{J}_{E}=\mathbf{Q}-\boldsymbol{\Pi}(\mathbf{u}-\mathbf{v}),
$$

where

$$
\begin{aligned}
& \mathbf{Q}=\sum_{\alpha}\left\{\mathbf{h}_{\alpha}\left(\mathbf{f}_{\alpha} \cdot \mathbf{v}_{\alpha}^{\prime}+\dot{\varepsilon}_{\alpha}\right)+\mathbf{u}_{\alpha}^{\prime} E_{\alpha}^{\prime} \delta_{\alpha}\right\}, \quad \mathbf{u}=\boldsymbol{\varphi}_{W} / \varrho_{W}, \\
& \boldsymbol{\Pi}=\sum_{\alpha}\left\{\mathbf{h}_{\alpha} \otimes \mathbf{f}_{\alpha}+\mathbf{u}_{\alpha}^{\prime} \otimes \mathbf{p}_{\alpha}^{\prime} \delta_{\alpha}\right\}=\partial_{\mathbf{v}} \boldsymbol{\Phi}_{E}^{\circ}
\end{aligned}
$$

with

$$
\mathbf{p}_{\alpha}^{\prime}=m \mathbf{v}_{\alpha}^{\prime}, \quad \mathbf{v}_{\alpha}^{\prime}=\mathbf{v}_{\alpha}-\mathbf{v}, \quad \mathbf{u}_{\alpha}^{\prime}=\mathbf{u}_{\alpha}-\mathbf{u}, \quad E_{\alpha}^{\prime}=m\left(v_{\alpha}^{\prime} 2 / 2+\varepsilon_{\alpha}\right) .
$$

Whenever $\mathbf{u}_{\alpha} \equiv \mathbf{v}_{\alpha}$, the relation (31) reduces to the Irving-Kirkwood form [18,33,13] but, otherwise, involves asymmetric contributions $\mathbf{u}_{\alpha}^{\prime} \otimes \mathbf{p}_{\alpha}^{\prime}$ to the stress tensor in (5), which we recall is given by $\mathbf{T}=-\langle\boldsymbol{\Pi}\rangle$. Although not discussed here, an angular momentum balance would no doubt reveal similar asymmetries associated with (29).

With no attempt to derive it from the foregoing statistical mechanics, we consider a linear continuum model that illustrates some of the key issues.

\section{Non-local linear viscoelasticity}

Fourier methods have been proposed by several workers as a route to homogenization [21,22,30], and the Coleman-Noll theory of simple materials is tantamount to the physicist's "long-wavelength" limit. In non-local linear theories, which represent a variant on the Coleman-Noll theory [5], Fourier representations embody the notion of "wave-number dependent" transport coefficients [19,9], capturing the dispersive effects associated with higher-gradients. When extended to the time domain by means of the Laplace transform, one obtains a similar description of frequency effects in materials with memory. Hence, the transform

$$
\hat{\psi}(\mathbf{k}, s)=\hat{\psi}_{t}(\mathbf{k}, s)=\int_{V} \int_{0}^{\infty} e^{-l \mathbf{k} \cdot \mathbf{x}-s t^{\prime}} \psi\left(\mathbf{x}, t-t^{\prime}\right) d V(\mathbf{x}) d t^{\prime},
$$

provides a localized description in Fourier space $(\mathbf{k}, s)$ of a spatio-temporally delocalized field $\psi_{t}\left(\mathbf{x}^{\prime}, t^{\prime}\right)=\psi\left(\mathbf{x}^{\prime}, t-t^{\prime}\right), \mathbf{x}^{\prime} \in V$, $t^{\prime} \geqslant 0$. Accordingly, a causal, non-local and linear constitutive equation between two sets of tensor fields

$$
\boldsymbol{\Phi}(\mathbf{x}, t)=\left\{\boldsymbol{\varphi}^{(1)}, \ldots, \boldsymbol{\varphi}^{(m)}\right\}(\mathbf{x}, t) \text { and } \boldsymbol{\Psi}(\mathbf{x}, t)=\left\{\boldsymbol{\psi}^{(1)}, \ldots, \boldsymbol{\psi}^{(m)}\right\}\left(\mathbf{x}, t-t^{\prime}\right),
$$

for $t^{\prime} \geqslant 0$, of the type studied extensively by Eringen [7,8], can be represented by the linear form:

$$
\widehat{\boldsymbol{\Phi}}(\mathbf{k}, s)=\widehat{\mathcal{L}}(\mathbf{k}, s) \widehat{\boldsymbol{\Psi}}(\mathbf{k}, s),
$$

where $\widehat{\mathcal{L}}(\mathbf{k}, s)$ represents a matrix of tensor moduli. When $\mathbf{\Phi}(\mathbf{x}, t)$ in (33) consists of stress and heat flux, one obtains a linear theory of thermo-viscoelasticity.

If we adopt a scaling in which $\mathbf{k}$ and $s$ are replaced by nondimensional forms $\lambda \mathbf{k}$ and $\tau s$, with $\lambda$ and $\tau$ denoting appropriate material length and time scales, then $k:=|\mathbf{k}|$ and $s$ represent, respectively, a Knudsen and a Deborah number. Hence, one obtain a weakly non-local spatio-temporal model from a Taylor series expansion of $\widehat{\mathcal{L}}(\mathbf{k}, s)$ about the spatially uniform rest-state $k=0, s=0$.

Loosely speaking, the expansion in $k$ is tantamount to the Burnett expansion of kinetic theory [26,1,2], whereas the expansion in $s$ represents the "retarded-motions" of Coleman and Noll [5,4]. In particular, the theory of simple fluids emerges at $O(k)$, while dissipative response arises at $O(s)$ for $s \rightarrow 0$; and, taken together, these approximations represent the NavierStokes-Fourier régime for fluids.

\subsection{Fluids}

To make the above points clearer, we consider a linear viscoelastic fluid in which stress relative to an equilibrium pressure $\mathbf{T}^{\prime}=\mathbf{T}+p_{\text {eq }} \mathbf{1}$ and heat flux $\mathbf{q}$, are represented by $\widehat{\mathbf{T}}^{\prime}\left[=\hat{\tau}_{i j}\right]$ and $\hat{\mathbf{q}}\left[=\hat{q}_{i}\right]$ as functions of velocity $\hat{\mathbf{v}}\left[=\hat{v}_{i}\right]$ and absolute temperature $\hat{\theta}$. Here as below the Cartesian-tensor conventions are employed for clarity, and (34) becomes: 


$$
\begin{aligned}
& \hat{q}_{i}=\widehat{L}_{i}^{(11)} \hat{\theta}+\widehat{L}_{i j}^{(12)} \hat{v}_{j}, \\
& \hat{\tau}_{i j}=\widehat{L}_{i j}^{(21)} \hat{\theta}+\widehat{L}_{i j l}^{(22)} \hat{v}_{l},
\end{aligned}
$$

where the coefficients $\widehat{L}$ depend on the complex frequency $s$ and wave vector $k_{i}$. For isotropic materials, these coefficients must be isotropic functions of the wave vector and, for the case of symmetric stress, can be written down explicitly as:

$$
\begin{aligned}
& \widehat{L}_{i}^{(11)}=A k_{i}, \quad \widehat{L}_{i j}^{(12)}=B \delta_{i j}+C k_{i} k_{j}, \quad \widehat{L}_{i j}^{(21)}=D \delta_{i j}+E k_{i} k_{j}, \\
& \widehat{L}_{i j l}^{(22)}=F \delta_{i j} k_{l}+G\left(\delta_{i l} k_{j}+\delta_{j l} k_{i}\right)+H k_{i} k_{j} k_{l},
\end{aligned}
$$

where the scalar coefficients $A, B, \ldots, H$ are assumed to be analytic functions of $s$ and $k^{2}=k_{i} k_{i}$. The assumption of Onsager symmetry would dictate that $B=D^{*}, C=E^{*}$, where asterisks denote complex conjugates.

Note that if $\hat{\tau}_{i j}$ is strictly dissipative, then the total dissipation is given (by Parseval's theorem) as the imaginary part of the integral over Fourier space of

$$
-\hat{q}_{i} k_{i} \hat{\theta}^{*}+\hat{\tau}_{i j} k_{i} \hat{v}_{j}^{*}=-\widehat{L}_{i}^{(11)} k_{i}|\hat{\theta}|^{2}-\widehat{L}_{i j}^{(12)} k_{i} \hat{v}_{j} \hat{\theta}^{*}+\widehat{L}_{i j}^{(21)} k_{i} \hat{v}_{j}^{*} \hat{\theta}+\widehat{L}_{i j l}^{(22)} k_{i} \hat{v}_{j}^{*} \hat{v}_{l}
$$

It is further worth noting that Onsager symmetry eliminates the energetic coupling of temperature and velocity gradients represented by the terms in $\widehat{L}_{i j}^{(a b)}, a \neq b$.

A weakly non-local theory in space is obtained from (36) by wave-number expansions of $K=A, B, \ldots, H$ of the form

$$
K=K_{0}(s)+K_{1}(s) k^{2}+K_{2}(s) k^{4} \ldots,
$$

where $K_{m}(s)$ are independent of $k_{i}$.

To compare with models proposed elsewhere [26,2], we retain of terms $O\left(k^{3}\right)$ in (36), to give

$$
\begin{aligned}
& \widehat{L}_{i}^{(11)}=\left(A_{0}+A_{1} k^{2}\right) k_{i}, \quad \widehat{L}_{i j}^{(12)}=\left(B_{0}+B_{1} k^{2}\right) \delta_{i j}+C_{0} k_{i} k_{j}, \\
& \widehat{L}_{i j}^{(21)}=\left(D_{0}+D_{1} k^{2}\right) \delta_{i j}+E_{0} k_{i} k_{j}, \\
& \widehat{L}_{i j l}^{(22)}=\left(F_{0}+F_{1} k^{2}\right) k_{l} \delta_{i j}+\left(G_{0}+G_{1} k^{2}\right)\left(\delta_{i l} k_{j}+\delta_{j l} k_{i}\right)+H_{0} k_{i} k_{j} k_{l},
\end{aligned}
$$

Galilean invariance implies that $B_{0}=0$, and Onsager symmetry further requires that $D_{0}=0$.

\subsection{Comparison to specific models}

On replacing $k_{l}$ by $\imath \nabla_{l}$ and $k^{2}$ by $-\nabla^{2}$ one obtains the corresponding spatial representation, with the coefficients $A_{0}(s), A(s), \ldots, H_{0}(s)$ now representing Laplace transforms of hereditary kernels. Thus, at this level, one captures (MaxwellCattaneo) relaxation of stress and heat conduction [24], in a form like that proposed by Müller [26].

It is worth noting that retention of terms of $O\left(k^{2}\right)$ in (39), together with imposition of Onsager symmetry, leads to eight coefficients

$$
A_{0}, B_{1}, C_{0}, D_{1}, E_{0}, F_{0}, G_{0}, H_{0},
$$

the same number as in the extension of kinetic theory proposed by Müller [26, Eq. (2.13)] (namely, his $a, b, c, K, L, \kappa, \lambda, \mu$ ), although the two formulae can be compared precisely only by a retarded-motion expansion to terms $O(s)$, with velocity $\hat{\mathbf{v}}$ and displacement $\hat{\mathbf{u}}$ connected by $\hat{\mathbf{v}}=s \hat{\mathbf{u}}$.

By contrast, Brenner's [1,2] models, discussed further below, are restricted to $O(s)$ in $s$ for $s \rightarrow 0$, and appear to be subsumed in the above-cited model of Müller. At this order, one obtains Maxwell's [25] theory of thermal stress from:

$$
\hat{\tau}_{i j}=F_{0}(0) \delta_{i j} k_{l} \hat{v}_{l}+G_{0}(0)\left(k_{i} \hat{v}_{j}+\hat{v}_{i} k_{j}\right)+\left[D_{1}(0) k^{2} \delta_{i j}+E_{0}(0) k_{i} k_{j}\right] \hat{\theta}
$$

with the terms in $F$ and $G$ representing viscous stress. We recall that Maxwell's theory gives the ratio $E_{0}(0) / G_{0}(0)$ as $3 v / \theta_{\text {eq }}$, where $v$ is kinematic viscosity. While no length scale is immediately apparent in this interpretation of (41), one sees that, by writing $\hat{v}_{i}=s \hat{u}_{i}$, the relevant terms in (41) become

$$
\tau s\left(k_{i} \hat{u}_{j}+\hat{u}_{i} k_{j}\right)+\lambda^{2} k_{i} k_{j} \hat{\theta} / \theta_{\mathrm{eq}}
$$

where we have identified the kinematic viscosity with the kinetic theory form $\lambda^{2} / 3 \tau$, in which $\lambda$ represents mean-free path and $\tau$ collisional relaxation time. Hence, with the (Maxwellian) view of viscosity as elastic relaxation, and with Newtonian viscosity given by the product of $\tau$ and a relevant elastic modulus (for gases, essentially the pressure), viscous effects of $O(s)$ (Deborah number) must be compared to thermal stresses of $O\left(k^{2}\right)$ (Knudsen number squared). ${ }^{14}$

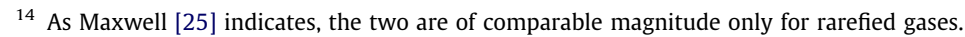


The preceding result is also captured by the bi-velocity hydrodynamics of Brenner [2], a recent form of which involves constitutive equations for entropy flux, relative velocity and stress ${ }^{15}$ :

$$
\begin{aligned}
& \mathbf{j}_{S}=-\kappa \nabla \ln \theta+\frac{1}{\theta} \Lambda_{12} \nabla \cdot \mathbf{T}^{\prime}, \\
& \mathbf{u}-\mathbf{v}=-\Lambda_{21} \nabla \ln \theta+\Lambda_{22} \nabla \cdot \mathbf{T}^{\prime}, \\
& \mathbf{T}^{\prime}=2 \mu\left[\nabla \mathbf{u}+(\nabla \mathbf{u})^{\mathrm{T}}\right]+\lambda(\nabla \cdot \mathbf{u}) \mathbf{1},
\end{aligned}
$$

with

$$
\mathbf{T}^{\prime}=\mathbf{T}+p_{\text {eq }} \mathbf{1}, \quad \text { and } \quad \mathbf{j}^{(S)}=\frac{1}{\theta}\left[\mathbf{q}+p_{\text {eq }}(\mathbf{u}-\mathbf{v})\right]
$$

where $p_{\text {eq }}$ is an equilibrium pressure, and $\mathbf{T}^{\prime}$ is the stress represented in (35). Hence, Brenner's theory substitutes an alternative velocity $\mathbf{u}$ (not to be confused with the displacement introduced above) for barycentric velocity $\mathbf{v}$ in the standard expression for viscous stress. Transforming (42) to Fourier space, it is a simple algebraic matter to show that it is a special case of (35). Without pursuing the issue further, the smaller number of coefficients in (42) than in (40) suggests that (42) may not capture all gradient effects at ("Burnett") order $k^{2}$.

\subsection{Other variables and effects}

The velocity and temperature fields are treated as primary independent variables in (35), in keeping with the standard continuum thermo-mechanics [4]. On the other hand, theories such as Eu's [9], involve gradients of mass density $\rho(\mathbf{x}, t)$, which in the formulation (35) translates to $\mathbf{k} \hat{\rho}(\mathbf{k}, s)$. However, the standard continuum mechanics gives density and the continuity equation in terms of (the Jacobian of) the material map between states, which in the above linear theory takes the form

$$
\widehat{\rho}^{\prime}=-\frac{1}{S} \rho_{\mathrm{eq}} \mathbf{k} \cdot \hat{\mathbf{v}}, \quad \text { where } \rho^{\prime}=\rho-\rho_{\mathrm{eq}},
$$

Hence, density gradients can be eliminated in favor of velocity gradients, introducing a simple pole at $s=0$ (with retarded motion given by Laurent rather than Taylor series). However, such singularities generally represent differential equations in $\partial_{t}$ that are common to the Maxwell-Cattaneo forms [26] of extended thermodynamics. ${ }^{16}$

Entropy has not been considered as a variable in the present discussion, which has largely avoided issues relating to the entropy balance and the associated thermodynamic restrictions on constitutive equations. It is clear that the higher-gradient effects discussed above involve departures of entropy flux $\mathbf{j}_{S}$ from the conventional form, which is evident in the bi-velocity model of Brenner and the extended thermodynamics of Müller. To the extent that this difference has implications for constitutive modeling, it is worthy of further investigation, including a consideration of Onsager symmetry and the restrictions on the non-local form (37).

A somewhat more compelling question is the stress asymmetry and couple-stress associated with non-barycentric forces and velocities, which was touched on in the above discussion of statistical mechanics. We recall that asymmetry $\mathbf{T} \neq \mathbf{T}^{\mathrm{T}}$ goes hand-in-hand with a couple-stress $\mathbf{M}\left[=\mu_{i j}\right]$ transmitting contact couple $\mathbf{M} d \mathbf{a}$ across areas da $=\mathbf{n d A}$. Because of the small length scales, such effects are expected to be relatively weak in the molecular system, other than in liquid crystals or in experiments involving high-frequency (large $k, s$ ) response. The situation is different at larger particle scales in fields such as granular mechanics and rotational seismology $[20,35,17,14]$, where gradient effects can be much more important.

Setting aside non-linear (amplitude) effects, the non-local model of viscoelasticity (34), which is also applicable to anisotropic materials, is readily modified to incorporate couple-stress effects. The isotropic versions (35) and (36) are readily modified by replacing $G\left(\delta_{i l} k_{j}+\delta_{j l} k_{i}\right)$ by the asymmetric form $G \delta_{i l} k_{j}+J \delta_{j l} k_{i}$, involving two coefficients and then adding a third row to (35) to specify $\mu_{i j}$ by means of new set of coefficients $F^{\prime}, G^{\prime}, H^{\prime}, J^{\prime}$.

It is worth noting that Cosserat models, endowed with independent micro-rotations $\omega\left[=\omega_{i}\right]$, provide an interesting alternative for the representation of micro-rotational inertia and couple-stress. A recently proposed complex-variable representation [14], with complex stress $\mathbf{T}+1 \mathbf{M}$ and complex velocity $\mathbf{v}+1 \omega$ giving a single balance for linear and angular momentum, is readily implemented in (35) and (36) on replacing $\hat{\tau}_{i j}$ by $\hat{\tau}_{i j}+\imath \hat{\mu}_{i j}, \hat{v}_{k}$ by $\hat{v}_{k}+\imath \hat{\omega}_{k}$ and $A, B, \ldots, J$ by complex coefficients. The resulting model also applies to couple-stress models without Cosserat rotation, obtained by taking $\omega \equiv 0$. This general subject is worthy of further investigation by means of an appropriate modification of the statistical mechanics discussed above, for which simpler versions have been applied to granular media [20, and references therein].

\footnotetext{
15 Brenner denotes $p_{\text {eq }}, \mathbf{T}^{\prime}, \mathbf{q}, \mathbf{u}-\mathbf{v}, \mathbf{j}_{s}$, respectively, by $p,-\mathbf{j}_{\mathbf{M}}, \mathbf{j}_{u}, \mathbf{j}_{w}, \mathbf{q}$, and employs a slightly different notation for constitutive coefficients.

16 In non-linear theories, we recall that the corresponding retarded-motion expansion can exhibit rest-state instability [10,26].
} 


\section{Conclusions}

The main findings and conclusions are summarized in the Abstract. It is worth re-iterating that the physical ambiguity of material points or material velocities in continuum mechanics is related to the breakdown of the simple-continuum model and the effects of higher spatial gradients in continuum fields. Several of the works cited above also address the effect of temporal fluctuations, and further investigations should be carried out to clarify the relative importance and relation between these effects. The present work shows that Galilean invariance allows for a rather direct derivation of continuum stress and heat flux from the statistical mechanical energy balance.

\section{Acknowledgements}

I wish to acknowledge the privilege and pleasure of a forty-year acquaintance with Professor K. R. Rajagopal. Also, I should like to express thanks to Professor Howard Brenner for numerous stimulating discussions of the issues raised by his work cited in this paper.

\section{Appendix A. Flux relations}

A restricted form of relations (24) and (25) has been given previously [13], and a more general form, with corrections, ${ }^{17}$ is given here. Starting from (19), one finds that

$$
\partial_{t} P_{\mathbf{A}}=\sum_{\alpha}\left\{\dot{\mathbf{A}}_{\alpha} \delta\left(\mathbf{x}_{\alpha}-\mathbf{x}\right)-\mathbf{A}_{\alpha} \mathbf{u}_{\alpha} \cdot \nabla \delta\left(\mathbf{x}_{\alpha}-\mathbf{x}\right)\right\}=\sum_{\alpha} \dot{\mathbf{A}}_{\alpha} \delta\left(\mathbf{x}_{\alpha}-\mathbf{x}\right)-\nabla \cdot \sum_{\alpha} \mathbf{u}_{\alpha} \otimes \mathbf{A}_{\alpha} \delta\left(\mathbf{x}_{\alpha}-\mathbf{x}\right)
$$

the second term of which is shown in (24). To reduce the first term to the desired form, note that, given a set of tensors and scalar weights $\left\{\mathbf{B}_{\alpha}, w_{\alpha}\right\}$, we may write the former as

$$
\begin{aligned}
& \mathbf{B}_{\alpha}=\sum_{\beta} \mathbf{B}_{\alpha \beta}+w_{\alpha} \overline{\mathbf{B}}, \quad \text { where } \mathbf{B}_{\alpha \beta}=\frac{\mathbf{1}}{W}\left(\mathbf{B}_{\alpha} w_{\beta}-\mathbf{B}_{\beta} w_{\alpha}\right)=-\mathbf{B}_{\beta \alpha}, \\
& \overline{\mathbf{B}}=\frac{\mathbf{1}}{W} \sum_{\alpha} \mathbf{B}_{\alpha}, \quad \text { and } \quad W=\sum_{\alpha} w_{\alpha} .
\end{aligned}
$$

Therefore,

$$
\sum_{\alpha} \mathbf{B}_{\alpha} \delta\left(\mathbf{x}_{\alpha}-\mathbf{x}\right)=\sum_{\alpha} \sum_{\beta} \mathbf{B}_{\alpha \beta} \delta\left(\mathbf{x}_{\alpha}-\mathbf{x}\right)+\overline{\mathbf{B}} \sum_{\alpha} w_{\alpha} \delta\left(\mathbf{x}_{\alpha}-\mathbf{x}\right) .
$$

However, the first term can be written as

$$
\begin{aligned}
\sum_{\alpha} \sum_{\beta} \mathbf{B}_{\alpha \beta} \delta\left(\mathbf{x}_{\alpha}-\mathbf{x}\right) & =\sum_{\alpha} \sum_{\beta<\alpha} \mathbf{B}_{\alpha \beta}\left[\delta\left(\mathbf{x}_{\alpha}-\mathbf{x}\right)-\delta\left(\mathbf{x}_{\beta}-\mathbf{x}\right)\right]=-\nabla \cdot \sum_{\alpha} \sum_{\beta<\alpha} \int_{\mathbf{x}_{\alpha}}^{\mathbf{x}_{\beta}} \delta(\mathbf{y}-\mathbf{x}) d \mathbf{y} \otimes \mathbf{B}_{\alpha \beta} \\
& =-\nabla \cdot \sum_{\alpha} \sum_{\beta} w_{\beta} \int_{\mathbf{x}_{\alpha}}^{\mathbf{x}_{\beta}} \delta(\mathbf{y}-\mathbf{x}) d \mathbf{y} \otimes \mathbf{B}_{\alpha} .
\end{aligned}
$$

The first term of (24) follows on taking $\mathbf{B}_{\alpha}=\dot{\mathbf{A}}_{\alpha}$.

The above derivation is somewhat easier in Fourier space $[13,30,9]$, where

$$
\delta(\mathbf{y}-\mathbf{x}) \rightarrow e^{-\mathbf{k} \cdot \mathbf{y}}, \nabla \rightarrow \imath \mathbf{k} .
$$

Moreover, in this representation

$$
\int_{\mathbf{x}_{\alpha}}^{\mathbf{x}_{\beta}} \delta(\mathbf{y}-\mathbf{x}) d \mathbf{y} \rightarrow \int_{\mathbf{x}_{\alpha}}^{\mathbf{x}_{\beta}} e^{-l \mathbf{k} \cdot \mathbf{y}} d \mathbf{y}=\left(\mathbf{x}_{\alpha}-\mathbf{x}_{\beta}\right)-\imath \mathbf{k} \cdot \int_{\mathbf{x}_{\alpha}}^{\mathbf{x}_{\beta}} \mathbf{y} \otimes d \mathbf{y}+O\left(|\mathbf{k}|^{2}\right)
$$

the first term of which leads to the localization [13] that defines simple materials and associated fluxes, such as Cauchy stress, with successively higher terms in $\mathbf{k}$ leading to higher-gradient theories of the type considered in Section 4.

\section{References}

[1] H. Brenner, Bi-velocity hydrodynamics: single-component fluids, IJES 47 (9) (2009) 930-958.

[2] H. Brenner, Bi-velocity transport processes. Single-component liquid and gaseous continua, Physica A 389 (7) (2010) $1297-1316$.

[3] A.L. Cauchy, De la pression ou tension dans un système de points matériels, Exercises de mathématique (1828), in: Ouvres complètes d'Augustin Cauchy, Ile Série, vol. 8, Gauthier-Villars, Paris, 1890, pp. 253-77.

[4] B.D. Coleman, Thermodynamics of materials with memory, Arch. Ration. Mech. Anal. 17 (1) (1964) 1-46.

[5] B.D. Coleman, W. Noll, Foundations of linear viscoelasticity, Rev. Mod. Phys. 33 (2) (1961) 239-249.

$\overline{17}$ In [13], the term $\sum_{i} \dot{\mathbf{A}}_{i} \delta(\overline{\mathbf{x}}-\mathbf{x})$ in Eq. (26) should read $\dot{\mathbf{A}} \sum_{i} m_{i} \delta\left(\mathbf{x}_{i}-\mathbf{x}\right) / m$ while $\sum_{i} \mathbf{x}_{i}$ in Eq. (27) should read $\sum_{i} m_{i} \mathbf{x}_{i}$. 
[6] M. Epstein, R. Segev, Differentiable manifolds and the principle of virtual work in continuum mechanics, J. Math. Phys. 21 (5) (1980) $1243-1245$.

[7] A.C. Eringen, Vistas of nonlocal continuum physics, IJES 30 (10) (1992) 1551-1565.

[8] A.C. Eringen, Nonlocal Continuum Field Theories, Springer, New York, 2002.

[9] B.C. Eu, Molecular theory of barycentric velocity: monatomic fluids, J. Chem. Phys. 128 (20) (2008) 204507-204510.

[10] R.L. Fosdick, K.R. Rajagopal, Thermodynamics and stability of fluids of third grade, Proc. Roy. Soc. A 369 (1738) (1980) $351-377$.

[11] P. Germain, The method of virtual power in continuum mechanics. Part 2: Microstructure, SIAM J. Appl. Math. 25 (3) (1973) $556-575$.

[12] J.D. Goddard, Microstructural origins of continuum stress fields - A brief history and some unresolved issues, in: D. De Kee, P.N. Kaloni (Eds.), Recent Developments in Structured Continua, Pitman Research Notes in Mathematics, vol. 143, Longman/John Wiley, New York, 1986, pp. 179-208.

[13] J.D. Goddard, Continuum modeling of granular assemblies, in: H.J. Herrmann et al. (Eds.), NATO ASI: Physics of Dry Granular Media, Kluwer, Dordrecht, 1998, pp. 1-24.

[14] J.D. Goddard, Granular hypoplasticity with cosserat effects, in: J.D. Goddard et al., (Eds.), IUTAM-ISIMM Symposium: Mathematical Modeling and Physical Instances of Granular Flows, AIP Conference Proceedings, AIP, New York, 2010, pp. 323-332.

[15] I. Goldhirsch, On the coarse graining of grains, in: J.D. Goddard et al., (Eds.), IUTAM-ISIMM Symposium: Mathematical Modeling and Physical Instances of Granular Flows, AIP Conference Proceedings, AIP, New York, 2010, pp. 198-207.

[16] A.E. Green, P.M. Naghdi, A unified procedure for construction of theories of deformable media. 1. Classical continuum physics \& 2. Generalized continua, Proc. Roy. Soc. London A 448 (1934). 335-356, 357-377.

[17] E.F. Grekova, W.H.K. Lee, Suggested readings in continuum mechanics and earthquake seismology, Bull. Seismol. Soc. Am. 99 (2B) (2009) $1076-1081$.

[18] J.H. Irving, J.G. Kirkwood, The statistical mechanical theory of transport properties IV. The equations of hydrodynamics, J. Chem. Phys. 18 (1950) 817.

[19] T. Keyes, I. Oppenheim, Bilinear hydrodynamics and the Stokes-Einstein law, Phys. Rev. A 8 (2) (1973) 937.

[20] N.P. Kruyt, Three-dimensional lattice-based dispersion relations for granular materials, in: J.D. Goddard et al., (Eds.), IUTAM-ISIMM Symposium: Mathematical Modeling and Physical Instances of Granular Flows, AIP Conference Proceedings, AIP, New York, 2010, pp. 405-415.

[21] I.A. Kunin, Elastic Media with Microstructure, Series in Solid-State Sciences, vol. 26, Springer-Verlag, Berlin, New York, 1982/1983.

[22] I.A. Kunin, On foundations of the theory of elastic media with microstructure, IJES 22 (8-10) (1984) 969-978.

[23] H. Lamb, Hydrodynamics, Cambridge University Press, Cambridge, UK, 1916.

[24] G.A. Maugin, The thermomechanics of nonlinear irreversible behaviors: An introduction, Series A, on Nonlinear Science, 27, World Scientific, Singapore, River Edge, NJ, 1999.

[25] J.C. Maxwell, Stresses in rarefied gases arising from inequalities of temperature, Philos. Trans. 170 (1879) 231-256.

[26] I. Müller, Rational Extended Thermodynamics, Springer, New York, 1998.

[27] A.I. Murdoch, A corpuscular approach to continuum mechanics: basic considerations, Arch. Ration. Mech. Anal. 88 (4) (1985) $291-321$.

[28] A.I. Murdoch, Objectivity in classical continuum physics: a rationale for discarding the 'principle of invariance under superposed rigid body motions' in favour of purely objective considerations, Cont. Mech. Thermo. 15 (3) (2003) 309-320.

[29] A.I. Murdoch, D. Bedeaux, On the physical interpretation of fields in continuum mechanics, IJES 31 (10) (1993) 1345-1373.

[30] A.I. Murdoch, D. Bedeaux, Characterization of microstates for confined systems and associated scale-dependent continuum fields via fourier coefficients, J. Phys. A 34 (33) (2001) 6495-6508.

[31] I.A. Murdoch, A critique of atomistic definitions of the stress tensor, J. Elast. 88 (2) (2007) 113-140.

[32] E. Nelson, Derivation of the Schrödinger equation from Newtonian mechanics, Phys. Rev. 150 (4) (1966) 1079.

[33] W. Noll, Die Herleitung der Grundgleichungen der Thermomechanik der Kontinua aus der statistischen Mechanik, J. Ration. Mech. Anal. 4 (1955) 627646. English Translation: R. Lehoucq, A. Von Lillenfeld-Toal, J. Elasticity, 100 (1) (2010) 5-24.

[34] P. Schofield, J.R. Henderson, Statistical mechanics of inhomogeneous fluids, Proc. Roy. Soc. A 379 (1776) (1982) 231-246.

[35] R. Teisseyre, Tutorial on new developments in the physics of rotational motions, Bull. Seismol. Soc. Am. 99 (2B) (2009) 1028-1039.

[36] C. Truesdell, W. Noll, The non-linear field theories of mechanics, in: S. Flügge (Ed.), Encyclopedia of Physics (Handbuch der Physik), vol. III/3, SpringerVerlag, Berlin, New York, 1965.

[37] C. Truesdell, K.R. Rajagopal, An Introduction to the Mechanics of Fluids, Birkhauser, Boston, 2000.

[38] C. Truesdell, R.A. Toupin, Principles of classical mechanics and field theory, in: S. Flügge (Ed.), Encyclopedia of Physics (Handbuch der Physik), vol. III/1, Springer, Berlin, 1960. 